Sir,

\section{Uveitis and Fabry disease}

In the recent article by Shen $e t \mathrm{al}^{1}$ entitled 'Fabry disease manifesting as chronic uveitis-treated with enzyme replacement therapy' the provocative presumption is that uveitis in a patient with Fabry disease was improved by enzyme replacement therapy. We have had experience with chronic uveitis in two patients $(0.4 \%$ of 527 patients in our clinic) with another lysosomal storage disease, Gaucher disease, ${ }^{2}$ but we did not see any improvement with specific replacement therapy, despite concern to the contrary. ${ }^{3}$ Both patients have otherwise mild Gaucher disease, but one patient has uveitis wellcontrolled for 12 years solely by local steroids whereas the other patient has suffered progression of uveitis despite 8 years of steroid drops followed by nearly 5 years of enzyme replacement therapy. The patient described by Shen $e t$ al arrived with best-corrected visual acuity (BCVA) at 20/400 (OD), and 20/200 (OS) which improved to 20/70 (OD), and 20/100 (OS) with steroid injection but then deteriorated. Enzyme replacement therapy for 24 weeks resulted in some apparent improvement, but required pars plana vitrectomy with resulting corneal opacity that left the patient with BCVA at 20/400 (OD) and 20/800 (OS) despite continued enzyme therapy.

Whereas the authors' hypothesis that specific 'lipidclearing' therapy should improve the condition if it is Fabry-related, we question whether uveitis in Fabry disease, as in Gaucher disease, is indeed related. In that this is a first report of concordance of uveitis and Fabry disease, heightened awareness of unusual ocular manifestations in Fabry disease is warranted. Nonetheless, despite our lack of optimism vis a vis uveitis, we hope that the patient will continue to benefit from enzyme therapy for all the classic disease parameters of Fabry disease, particularly cardiac manifestations ${ }^{4}$ which were not mentioned in the report.

\section{References}

1 Shen YD, Yang CM, Huang JS. Fabry disease manifesting as chronic uveitis-treated with enzyme replacement therapy. Eye 2006, 14 July [E-pub ahead of print].

2 Dweck A, Wollstein G, Elstein D, Zimran A. Uveitis in Gaucher disease: a case report. Am J Ophthalmol 2005; 140: 146-147.

3 Dweck A, Zimran A, Elstein D. Reply. Am J Ophthalmol 2006; 141: 421-422.

4 Altarescu G, Elstein D. Cardiac abnormalities in Fabry disease: natural history in hemizygote males suggests that cardiac pathology is universally present. Haema 2004; 8: 103-108.
D Elstein, G Altarescu and A Zimran

Shaare Zedek Medical Center, Gaucher Clinic, One Bazak Road, PO Box 3235, Jerusalem 91031, Israel

Correspondence: D Elstein,

Tel: + 972 6554123;

Fax: +9727660 .

E-mail: elstein@szmc.org.il

Eye (2007) 21, 448. doi:10.1038/sj.eye.6702625;

published online 6 October 2006

Sir,

Reply to Dweck et al

We would like to comment on the letter from Dweck et al. First, the presentations in this Fabry case was not typical of a true uveitis because it lacked some common features of a uveitis, such as injection of ocular surface, kerato precipitates of posterior corneal surface, or posterior synechia of iris, despite the anterior chamber cells were very prominent. We believe the change of vascular permeability contributed to the prominent cells in both anterior and posterior chamber and thus consider this case a uveitis-like picture rather than a true uveitis. Second, we agree with the speculation that the occurrence of uveitis in the Fabry case was a coincidence. However, because the clinical condition markedly improved after enzyme replacement therapy, this coincidence was not very likely.

Based on the experience of many vitreoretinal doctors on the use of corticosteroids in diabetic macular oedema, ${ }^{1,2}$ we believe the use of steroid in this case was similar to that in diabetic retinopathy: a vascular leakagedecreasing effect rather than a true anti-inflammatory effect. This explained why the effect of steroid was temporary and the enzyme replacement therapy had more long-term effect.

We believe the combined therapy of steroid and enzyme replacement therapy would be a reasonable approach to cases with Fabry disease or Gaucher disease.

\section{References}

1 Bakri SJ, Kaiser PK. Posterior subtenon triamcinolone acetonide for refractory diabetic macular edema. Am J Ophthalmol 2005; 139(2): 290-294.

2 Karacorlu M, Ozdemir H, Karacorlu S, Alacali N, Mudun B, Burumcek E. Intravitreal triamcinolone as a primary therapy in diabetic macular oedema. Eye 2005; 19(4): 382-386. 
Y-D Shen ${ }^{1,2}$

${ }^{1}$ Department of Ophthalmology, Changhua

Christian Hospital, Changhua, Taiwan

${ }^{2}$ Department of Ophthalmology, National Taiwan

University Hospital, Taipei, Taiwan

Correspondence: Y-D Shen,

Department of Ophthalmology,

National Taiwan University Hospital,

7, Chung-Shan S Road,

Taipei 100, Taiwan

Tel: + 886223123456 ext. 5205;

Fax: + 886223412875

E-mail: ydshen@ha.mc.ntu.edu.tw

Eye (2007) 21, 448-449. doi:10.1038/sj.eye.6702627;

published online 6 October 2006

Sir,

\section{Patient safety in cataract surgery}

We thank Dr Pandey and Mr Scott for their interest in our article. We support their observations drawing attention to the danger of incorrectly mounted Luer lock intraocular cannulae as a potential risk. Following notification of this case report, the National Reporting and Learning (NRLS) database at the National Patient Safety Agency (NPSA) was searched for any similar patient safety incidents. On the NRLS database, three more similar incidents and one near miss were retrieved. These cases along with the example reported by Pandey and Scott share a common consequential theme. This is of intraocular cannulae - such as Rycroft, hydro-dissection, or wound hydration cannulae - 'exploding' from syringes during cataract surgery. While root cause of events is not confirmed, failure to correctly engage Luer lock cannulae appears to be an issue. As such it might be best considered as 'user error' and is a patient safety incident, rather than a device failure. It is prudent to highlight device incidents to the Medicines and Healthcare products Regulatory Agency (MHRA) so that any device products concerned might be investigated further. We have now brought this matter to their attention.

We support the publicity of patent safety incidents so that the learning arising and any solutions emerging can be shared. Ophthalmic staff should be aware of this specific risk with mounting Luer lock syringes. We applaud the practice of Pandey and Scott of checking secure engagement of Luer lock mounted cannulae before their introduction into the eye. We commend the reporting of all patient safety incidents in both NHS and in independent sector care.

SP Kelly ${ }^{1}$ and NJ Astbury ${ }^{2}$

${ }^{1}$ Bolton Hospital NHS Trust, Lancashire, UK

${ }^{2}$ Norfolk and Norwich University Hospital NHS

Trust, Norwich, UK

Correspondence: SP Kelly, Bolton Hospital NHS Trust, Lancashire, UK

Tel: + 440120 4390694;

Fax: + 4401204390554

E-mail: spkelly@ntlworld.com

Eye (2007) 21, 449. doi:10.1038/sj.eye.6702626; published online 13 October 2006

Sir,

Locking the Luer lock

I read with interest the article on 'Patient safety in cataract surgery'. ${ }^{1}$ As stated in the article, penetration of the globe during periocular injections is rightly, a reportable incident. Complications however also occur with the use of syringes intracamerally. I would like to draw your attention to the case of an 85-year-old gentleman who underwent uneventful left phacoemulsification surgery. At the end of the procedure, a Luer lock syringe was used with a 27-gauge Rycroft cannula for stromal hydration around the corneal section. The cannula tip dislodged under pressure and penetrated the eye through the wound to cause a hyphaema, choroidal haemorrhage, and total retinal detachment.

He was managed initially with a vitrectomy, removal of the intraocular lens with drainage of the suprachoroidal haemorrhage, and silicone oil internal tamponade. He developed proliferative vitreoretinopathy with a perisilicone retinal detachment that was managed with a retinectomy and silicone oil top-up procedure 6 weeks later. The silicone oil was successfully removed 3 months later with secondary anterior chamber intraocular lens implantation; his final best-corrected visual acuity was $6 / 18$

Case reports of similar occurrences have been reported in the literature, one with the use of Luer locked syringe during hydrodissection ${ }^{2}$ and one with a nonthreaded syringe. ${ }^{3}$ 